\title{
Hipericão na perturbação depressiva: revisão crítica de autor
}

Tiago da Cunha Costa', Filipa Paraíso², Daniela Marafona Pereira ${ }^{3}$, Maria João Coelho ${ }^{4}$

\section{RESUMO}

Objetivos: Rever a evidência existente quanto à eficácia, tolerabilidade e segurança do hipericão no tratamento da perturbação depressiva (PD).

Fontes de dados: NICE, Canadian Medical Association Infobase, PubMed - Clinical Queries, The Cochrane Library, BMJ, Bandolier e DARE - Centre for Reviews and Dissemination.

Métodos: Foi realizada a pesquisa de artigos científicos em plataformas online de medicina baseada na evidência, com as palavras-chave Hypericum e Depressive Disorder. A seleção dos artigos foi feita com base no título, ano de publicação e resumo. Resultados: Foram incluídas nesta revisão duas normas de orientação clínica (NOC), três meta-análises (MA), duas revisões sistemáticas (RS) e um artigo original (AO). Uma NOC referiu que o hipericão pode ser usado como primeira linha na PD major ligeira a moderada e como tratamento adjuvante de segunda linha na PD major moderada a grave. A outra NOC não aconselha o seu uso. As MA e as RS concluíram que o hipericão apresenta uma eficácia superior ao placebo no tratamento da PD, sendo que duas MA e as RS acrescentaram que não há aumento dos efeitos adversos. As duas RS referiram o risco de interações com outros fármacos. O AO também afirma a eficácia do hipericão nas mulheres na pós-menopausa.

Conclusão: Existe evidência quanto à eficácia do hipericão na PD ligeira a moderada. Os dados disponíveis parecem ser consistentes quanto à sua boa tolerabilidade, mas também quanto ao risco de interações com outros fármacos. As limitações da recomendação prendem-se essencialmente com o facto dos mecanismos de ação não estarem completamente esclarecidos.

Palavras-chave: Hipericão; Perturbação depressiva.

\section{INTRODUÇÃO}

A perturbação depressiva (PD) é uma das patologias mais comuns em todo o mundo, ${ }^{1-2}$ estando a sua prevalência a aumentar. ${ }^{1}$ Em Portugal, a PD apresenta uma prevalência anual de 7,9\%. ${ }^{3}$ Esta condição tem um impacto significativo no dia-a-dia dos utentes, pelo que é importante o seu correto tratamento. ${ }^{1}$

O tratamento farmacológico apresenta um papel bem estabelecido. ${ }^{2}$ No entanto, o estigma quanto à sua eficácia, custos e efeitos colaterais leva muitos utentes

1. Médico Interno de Medicina Geral e Familiar. USF Hygeia, ACeS Tâmega III - Vale do Sousa Norte. Vila Cova da Lixa, Portugal.

2. Médica Interna de Medicina Geral e Familiar. USF Santa Luzia, ACeS Tâmega III Vale do Sousa Norte. Freamunde, Portugal.

3. Médica Interna de Medicina Geral e Familiar. USF Salvador Lordelo, ACeS Tâmega II - Vale do Sousa Sul. Lordelo, Portugal.

4. Médica Interna de Medicina Geral e Familiar. USF Freamunde, ACeS Tâmega III Vale do Sousa Norte. Freamunde, Portugal. a não aderirem a este tipo de tratamento e a procurar alternativas, como, por exemplo, o hipericão. ${ }^{1,4}$

O hipericão, também conhecido como erva de São João, é um suplemento nutricional proveniente da planta Hypericum perforatum, habitualmente adjetivado como «natural» e muito utilizado para o alívio da sintomatologia depressiva. ${ }^{5}$ Em Portugal, este suplemento é comercializado de forma livre em farmácias, ervanárias e lojas de produtos naturais, não sendo objeto de regulamentação pelo INFARMED. ${ }^{6}$ A sua principal substância ativa, a hipericina, está isoladamente associada a uma baixa incidência de efeitos adversos; ${ }^{7}$ no entanto, esta apresenta várias interações medicamentosas conhecidas ${ }^{8}$ pelo que o seu efeito benéfico não é consensual na literatura.

Esta revisão tem por objetivo rever a evidência existente quanto à eficácia, tolerabilidade e segurança do hipericão no tratamento da PD. 


\section{MÉTODOS}

Foi realizada uma pesquisa de artigos científicos publicados em plataformas online de medicina baseada na evidência. As plataformas utilizadas foram o $\mathrm{Na}$ tional Institute of Health and Care Excellence (NICE), Canadian Medical Association Infobase, PubMed - Clinical Queries, The Cochrane Library, The British Medical Journal (BMJ) - Evidence-Based Medicine, Bandolier e DARE - Centre for Reviews and Dissemination.

A pesquisa foi realizada em fevereiro de $2020 \mathrm{com}$ as palavras-chave Hypericum e Depressive Disorder.

Numa primeira fase foram pesquisadas normas de orientação clínica (NOC) e artigos de revisão [revisões sistemáticas (RS) e meta-análises (MA)] publicados a partir de 2010, inclusive. Numa fase posterior foram pesquisados estudos originais (EO) publicados a partir de 2015, inclusive. Os artigos foram incluídos por respeitarem o modelo PICO (Tabela 1) e por estarem redigidos nas línguas Portuguesa e Inglesa. Artigos em duplicado foram excluídos.

\section{RESULTADOS}

Na pesquisa efetuada obtiveram-se 13 NOC, 26 revisões e 16 EO. Após aplicação dos critérios de inclusão e exclusão foram incluídos nesta revisão duas NOC, cinco revisões (três MA e duas RS) e um EO. Os fluxogramas de seleção dos artigos podem ser consultados nas Figuras 1 e 2. O resumo dos artigos incluídos encontra-se descrito nas Tabelas 2, 3, 4 e 5.

\section{Normas de orientação clínica}

A NOC publicada em 2016 pela Canadian Network for Mood and Anxiety Treatments ${ }^{9}$ recomenda, na secção Complementary and Alternative Medicine Treatments, o uso do hipericão como primeira linha, em monoterapia, na PD major ligeira a moderada. Esta recomendação é baseada em duas RS que apresentaram resultados consistentes. Recomendam também que o hipericão pode ser usado como tratamento adjuvante de segunda linha na PD major moderada a grave, recomendação baseada em duas RS que apresentavam resultados contraditórios.
A NOC publicada pelo Scottish Intercollegiate Guidelines Network, ${ }^{10}$ em 2010, afirma não ser possível aconselhar o uso do hipericão na PD, uma vez que não existe uniformização de doses e existe risco de interação com outros fármacos, de que são exemplo os contracetivos orais. Esta recomendação é apoiada por uma RS de elevada qualidade publicada na Cochrane Libra$r y$.

\section{Meta-análises}

Apaydin e colaboradores ${ }^{1}$ concluíram que o hipericão em monoterapia tem uma eficácia superior ao placebo na PD major ligeira a moderada. Esta eficácia foi demonstrada, pois os utentes a fazer hipericão foram mais respondedores ao tratamento (diminuição autorreportada dos sintomas depressivos) e apresentavam valores médios inferiores nas escalas de depressão [e.g., Escala de avaliação de depressão de Hamilton (HAM-D)] comparativamente ao placebo. No entanto, o número de utentes em remissão no fim do follow-up dos estudos não foi significativamente diferente (estudos com follow-up de quatro a 12 semanas, mediana de seis semanas). Concluíram também que os efeitos adversos do hipericão são comparáveis aos do placebo (MA de 13 ensaios clínicos aleatorizados (ECA), $N=2.600$ ). Como limitações desta MA, de referir que os estudos incluídos apresentavam heterogeneidade, alguns de baixa qualidade, avaliaram um número limitado de potenciais efeitos adversos e não foram desenhados para avaliar efeitos adversos raros. Para além disso, existiam poucos estudos a comparar os diferentes extratos e doses do hipericão.

Linde e colaboradores ${ }^{2}$ demonstraram que o hipericão tem efeitos benéficos no tratamento da PD, pois na MA em rede de 66 ECA ( $N=15.161)$ mostrou ser mais eficaz (reduções $\geq 50 \%$ e resultado final inferior a um 


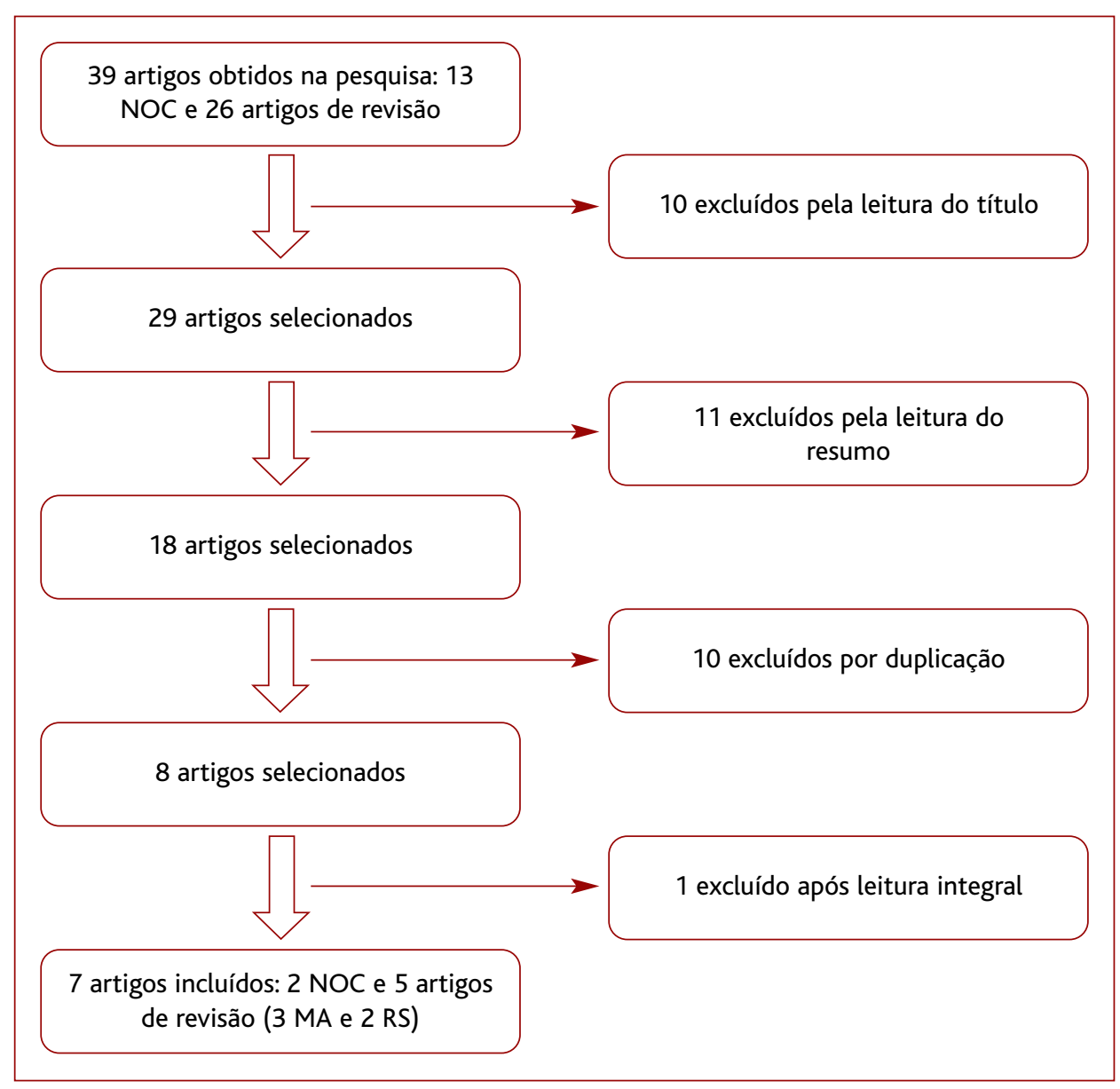

Figura 1. Fluxograma de seleção das normas de orientação clínica e artigos de revisão.

limite fixo nas escalas de depressão) e sem efeitos adversos diferentes do placebo. As principais limitações da MA são a inclusão de doentes com diferentes tipos de gravidade da PD e com outcomes avaliados em diferentes intervalos temporais (quatro, seis ou oito semanas).

Kasper e colaboradores ${ }^{4}$ concluíram que o extrato de hipericãoWS 5570, na dose diária de 900mg, é superior ao placebo na depressão major ligeira a moderada independentemente da faixa etária. Esta superioridade foi demonstrada pela maior percentagem de utentes que obtiveram boa resposta e que atingiram a remissão em relação ao placebo, diferenças com significância estatística (MA com $N=1.328$ ). Esta revisão apresentou como limitação o facto de o número de utentes incluídos no estudo com $\geq 60$ anos ser relativamente reduzi- do $(N=132)$, o que limita a evidência e as recomendações para este grupo etário.

\section{Revisões sistemáticas}

Sarris e colaboradores ${ }^{11}$ concluíram que existe boa evidência para o uso de hipericão na PD major. Esta conclusão baseou-se na maior eficácia demonstrada em duas MA, três RS e três ECA, apesar de outros dois ECA não o terem demonstrado. A nível da tolerabilidade, uma RS $(N=35.562)$ mostrou que o grau de efeitos adversos é comparável ao placebo. Quanto à segurança, uma RS, dois ECA e um estudo in vitro mostraram a existência de interações farmacológicas, como a redução da concentração plasmática de alguns fármacos. Esta revisão apresenta algumas limitações, nomeadamente a inconsistência dos estudos incluídos e 


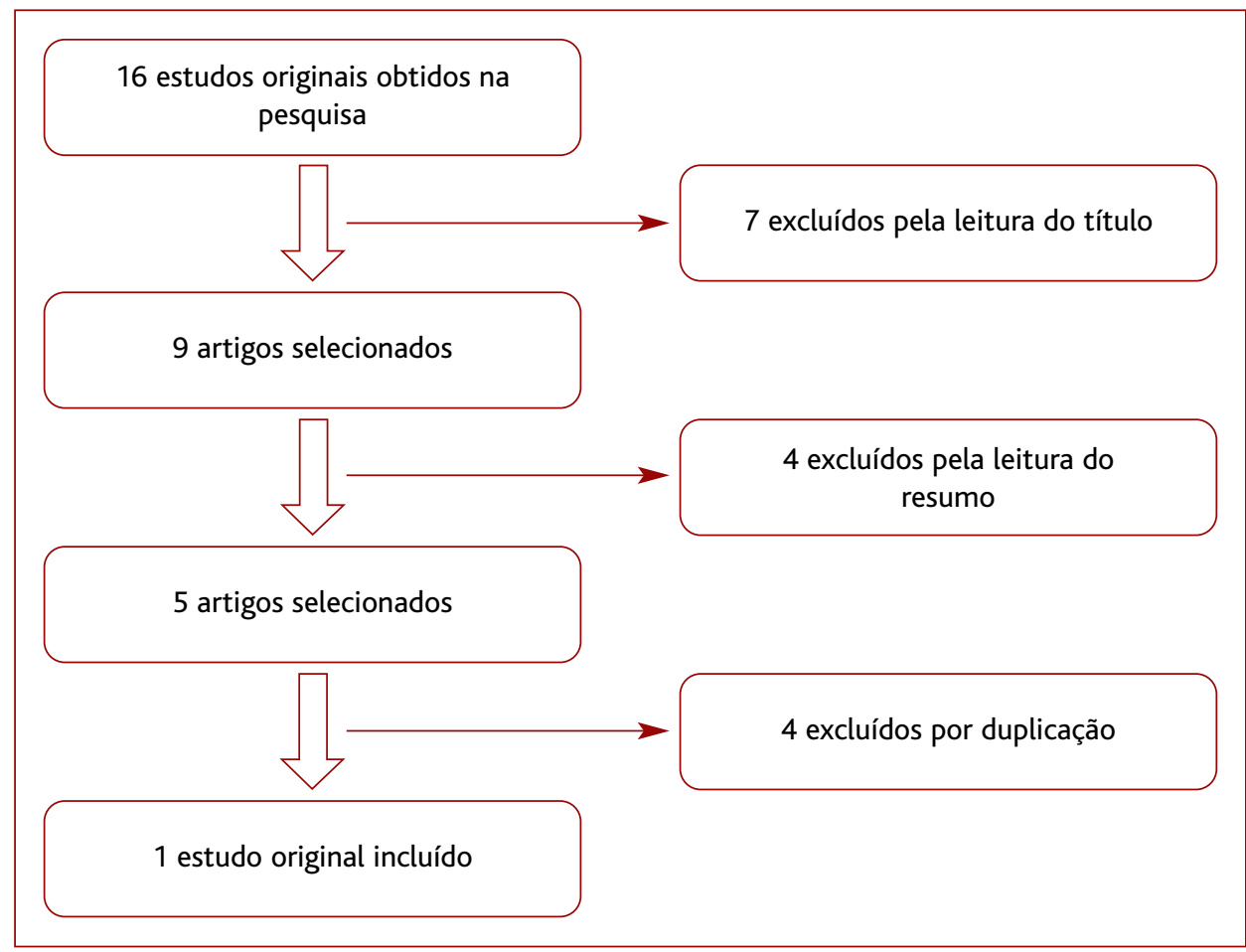

Figura 2. Fluxograma de seleção dos estudos originais.

\section{TABELA 2. Resumo das normas de orientação clínica.}

\section{Título}

Canadian Network for Mood and Anxiety Treatments (CANMAT) 2016 Clinical guidelines for the management of adults with major depressive disorder

Scottish Intercollegiate Guidelines Network: non-pharmaceutical management of depression in adults - a national clinical guideline

\section{Recomendações}

Recomendado como primeira linha, em monoterapia, na PD major ligeira a moderada.

Recomendado como tratamento adjuvante de segunda linha na PD major moderada a grave.

Não é possível aconselhar o seu uso na depressão, pois não existe uniformização da dose e existe risco de interações medicamentosas. o facto de não terem sido comparados os diferentes extratos e doses do hipericão.

Cipriani e colaboradores ${ }^{12}$ afirmaram que apenas existem benefícios minor do hipericão no tratamento da depressão major e que provavelmente não existe benefício na depressão de longa duração. Afirmaram também que não existe evidência robusta sobre a eficácia na depressão major grave. Os efeitos adversos do hipe- ricão são comparáveis aos do placebo (MA de 14 ECA). Quanto à segurança, os autores encontraram em duas RS evidência de interações farmacológicas, como por exemplo com fármacos antidepressivos. Esta RS incluiu estudos bastante heterogéneos, nomeadamente no que diz respeito às características das populações estudadas, instrumentos de avaliação de sintomas e preparações de hipericão utilizadas. 


\begin{tabular}{|c|c|c|c|}
\hline $\begin{array}{l}\text { Referência } \\
\text { bibliográfica }\end{array}$ & Resultados & Conclusões & Limitações \\
\hline $\begin{array}{l}\text { Apaydin et al. } \\
\text { (2016) }\end{array}$ & $\begin{array}{l}\text { Eficácia } \\
\text { Utentes mais respondedores [MA de } 18 \text { ECA } \\
\text { ( } N=2.922), R R=1,53 \text { para IC } 95 \% \text { de } 1,19 \text { a } \\
1,97 \text { ] e com valores médios inferiores nas } \\
\text { escalas de depressão [MA de } 16 \text { ECA } \\
\text { ( } N=2.888 \text { ), diferenças médias padronizadas } \\
=0,49 \text { para IC95\% de } 0,23 \text { a } 0,74 \text { ], resultados } \\
\text { estatisticamente significativos; } \\
\text { Número de utentes em remissão não foi } \\
\text { significativamente diferente [MA de } 9 \text { ECA } \\
\text { ( } N=1.419), \text { RR=1,69 para IC } 95 \% \text { de } 0,63 \text { a } \\
4,55, \text { mediana de follow-up de } 6 \text { semanas]. } \\
\text { Tolerabilidade } \\
\text { A MA de } 13 \text { ECA ( } N=2.600 \text { ) não mostrou } \\
\text { diferenças no número de doentes com efeitos } \\
\text { adversos (OR=0,83 para IC95\% de } 0,62 \text { a } 1,13 \text { ) } \\
\text { nem na existência de efeitos adversos graves } \\
\text { (OR=0,26 para IC95\% de } 0,04 \text { a } 1,23 \text { ), entre os } \\
\text { tratados com hipericão ou placebo; } \\
\text { Hipericão associado a mais efeitos adversos } \\
\text { neurológicos (OR=1,56 para IC95\% de } 1,08 \text { a } \\
\text { 3,32) quando comparado ao placebo. }\end{array}$ & $\begin{array}{l}\text { O hipericão em monoterapia } \\
\text { tem uma eficácia superior ao } \\
\text { placebo na PD major ligeira a } \\
\text { moderada. } \\
\text { Efeitos adversos comparáveis } \\
\text { aos do placebo. }\end{array}$ & $\begin{array}{l}\text { Estudos com } \\
\text { heterogeneidade e alguns } \\
\text { com baixa qualidade. } \\
\text { Poucos estudos a comparar } \\
\text { diferentes extratos e doses } \\
\text { do hipericão. } \\
\text { ECA avaliaram um número } \\
\text { limitado de potenciais } \\
\text { efeitos adversos e não foram } \\
\text { desenhados para avaliar } \\
\text { efeitos adversos raros. }\end{array}$ \\
\hline $\begin{array}{l}\text { Linde et al. } \\
\text { (2015) }\end{array}$ & $\begin{array}{l}\text { Eficácia } \\
\text { O hipericão foi significativamente superior ao } \\
\text { placebo [MA em rede de } 66 \text { ECA }(N=15.161) \text {, } \\
\text { OR=2,03 para IC95\% de } 1,63 \text { a } 2,53] \text {. } \\
\text { Tolerabilidade } \\
\text { Sem efeitos adversos significativamente } \\
\text { diferentes [MA em rede de } 66 \text { ECA }(N=15.161) \text {, } \\
\text { OR=1,04 para IC95\% de } 0,53 \text { a } 2,06] \text {. }\end{array}$ & $\begin{array}{l}\text { O hipericão tem efeitos } \\
\text { benéficos no tratamento da } \\
\text { PD. }\end{array}$ & $\begin{array}{l}\text { Incluídos na mesma análise } \\
\text { pacientes com diferentes } \\
\text { tipos de gravidade e } \\
\text { avaliados outcomes com } \\
\text { diferentes intervalos } \\
\text { temporais. } \\
\text { Não foram avaliados efeitos } \\
\text { a longo prazo. }\end{array}$ \\
\hline $\begin{array}{l}\text { Kasper et al. } \\
\text { (2015) }\end{array}$ & $\begin{array}{l}\text { Eficácia } \\
\text { Maior percentagem de utentes sob hipericão } \\
\text { com boa resposta (reduções } \geq 50 \% \text { na HAM-D } \\
\text { às } 6 \text { semanas) e em remissão (HAM-D } \leq 7 \\
\text { pontos às } 6 \text { semanas) em relação ao placebo, } \\
\text { diferenças estatisticamente significativas (MA } \\
\text { com } N=1.328 \text { ). Efeito comparável nos utentes } \\
\text { com } \geq 60 \text { anos e com }<60 \text { anos. }\end{array}$ & $\begin{array}{l}\text { O hipericão WS 5570, na } \\
\text { dose diária de 900mg, é } \\
\text { superior ao placebo na } \\
\text { depressão major ligeira a } \\
\text { moderada, } \\
\text { independentemente da faixa } \\
\text { etária. }\end{array}$ & $\begin{array}{l}\text { Número de utentes incluídos } \\
\text { com } \geq 60 \text { anos foi } \\
\text { relativamente reduzido. }\end{array}$ \\
\hline
\end{tabular}

\section{Estudos originais}

Eatemadnia e colaboradores, ${ }^{13}$ num ECA controlado duplamente-cego ( $N=70)$, mostraram que o tratamento com hipericão é eficaz na redução dos sintomas da 
TABELA 4. Resumo das revisões sistemáticas.

\begin{tabular}{|c|c|c|c|}
\hline $\begin{array}{l}\text { Referência } \\
\text { bibliográfica }\end{array}$ & Resultados & Conclusões & Limitações \\
\hline $\begin{array}{l}\text { Sarris et al. } \\
\text { (2017) }\end{array}$ & $\begin{array}{l}\text { Eficácia } \\
\text { Superioridade do hipericão em relação ao } \\
\text { placebo no tratamento da PD major ( } 2 \text { MA, } \\
3 \text { RS e } 3 \text { ECA); } \\
\text { Sem superioridade do hipericão em relação ao } \\
\text { placebo (2 ECA). } \\
\text { Tolerabilidade } \\
\text { O grau de efeitos adversos é comparável ao } \\
\text { placebo [(1 RS ( } N=35.562)] \text {. } \\
\text { Segurança } \\
\text { Existem interações do hipericão com outros } \\
\text { fármacos ( } 1 \text { RS, } 2 \text { ECA e } 1 \text { estudo in vitro). }\end{array}$ & $\begin{array}{l}\text { Existe boa evidência para o } \\
\text { uso de hipericão na PD major. }\end{array}$ & $\begin{array}{l}\text { Inconsistência nos estudos } \\
\text { incluídos. } \\
\text { Não foram comparados os } \\
\text { diferentes extratos e doses } \\
\text { do hipericão. }\end{array}$ \\
\hline $\begin{array}{l}\text { Cipriani et al. } \\
\text { (2011) }\end{array}$ & $\begin{array}{l}\text { Eficácia } \\
\text { O hipericão foi mais eficaz que o placebo na } \\
\text { melhoria da sintomatologia [(MA de } 18 \mathrm{ECA} \\
\text { ( } N=3.064), \mathrm{RR}=1,48 \text { para IC95\% de } 1,23 \text { a } 1,77] \\
\text { e no aumento da proporção de pessoas em } \\
\text { remissão [(MA de } 6 \text { ECA }(N=1.236), \mathrm{RR}=2,77 \\
\text { para IC95\% de 1,80 a 4,26)]. } \\
\text { Tolerabilidade } \\
\text { Sem diferença estatisticamente significativa } \\
\text { entre o hipericão e o placebo em relação aos } \\
\text { efeitos adversos [(MA de } 14 \text { ECA ( } N=2.496) \text {, } \\
\text { OR=0,98 para IC95\% de } 0,78 \text { a 1,23)]. } \\
\text { Segurança: } \\
\text { Evidência de interações significativas com } \\
\text { outros fármacos (2 RS). }\end{array}$ & $\begin{array}{l}\text { A melhor evidência mostra } \\
\text { benefícios minor na } \\
\text { depressão major e, } \\
\text { provavelmente, não existe } \\
\text { benefício na depressão de } \\
\text { longa duração. } \\
\text { Não existe evidência robusta } \\
\text { sobre a eficácia na depressão } \\
\text { major grave. }\end{array}$ & $\begin{array}{l}\text { Heterogeneidade dos } \\
\text { estudos (extratos e doses do } \\
\text { hipericão variáveis, } \\
\text { populações muito } \\
\text { heterogéneas e instrumentos } \\
\text { de avaliação de sintomas } \\
\text { variáveis). }\end{array}$ \\
\hline
\end{tabular}

depressão nas mulheres na pós-menopausa. Este estudo comparou o efeito da suplementação com hipericão na dose de 990mg/dia, durante oito semanas, em relação a um placebo. De facto, o grupo suplementado com hipericão apresentou uma redução estatisticamente significativa na sintomatologia da depressão (avaliada pela HAM-D). O estudo apresenta uma amostra reduzida e um tempo de follow-up inferior à dos outros estudos (< 12 semanas). Por outro lado, não é explicado se a melhoria dos sintomas depressivos ocorre direta- mente relacionada com a redução dos sintomas vasomotores ou se é uma melhoria independente.

\section{CONCLUSÃO}

O hipericão é uma planta medicinal que contém moléculas farmacologicamente ativas e com potencial terapêutico. ${ }^{14}$

São vários os possíveis mecanismos de ação, ainda que não completamente esclarecidos. Vários dos estudos incluídos ${ }^{4,11,13}$ afirmam que um dos mecanismos 


\begin{tabular}{|c|c|c|c|}
\hline $\begin{array}{l}\text { Referência } \\
\text { bibliográfica }\end{array}$ & Resultados & Conclusões & Limitações \\
\hline $\begin{array}{l}\text { Eatemadnia } \\
\text { et al. (2019) }\end{array}$ & $\begin{array}{l}\text { Eficácia } \\
80 \% \text { das mulheres no grupo de intervenção } \\
\text { (hipericão } 990 \mathrm{mg} / \text { dia, } N=35 \text { ) não apresentaram } \\
\text { depressão no fim do follow-up do estudo } \\
\text { (8 semanas) em comparação com } 5,7 \% \text { no } \\
\text { grupo placebo ( } N=35 \text { ), diferenças } \\
\text { estatisticamente significativas. }\end{array}$ & $\begin{array}{l}\text { O tratamento com hipericão } \\
\text { é uma maneira eficaz de } \\
\text { reduzir a depressão nas } \\
\text { mulheres na pós-menopausa. }\end{array}$ & $\begin{array}{l}n \text { do estudo relativamente } \\
\text { reduzido. } \\
\text { Follow-up reduzido } \\
\text { (< } 12 \text { semanas). } \\
\text { Não avaliam a relação entre } \\
\text { a melhoria dos sintomas } \\
\text { depressivos e a redução dos } \\
\text { sintomas vasomotores. }\end{array}$ \\
\hline
\end{tabular}

passa pela inibição não seletiva da recaptação de neurotransmissores (e.g., serotonina, dopamina e noradrenalina).

O presente estudo versou analisar o uso de hipericão como uma alternativa plausível quanto à eficácia, tolerabilidade e segurança nos utentes com PD que rejeitem os antidepressivos convencionais, motivo pelo qual se procedeu à comparação do uso do hipericão com o placebo.

A maioria dos estudos mostrou que existe evidência para a utilização do hipericão no tratamento da PD, ainda que não seja consensual qual a posologia, duração e extrato mais adequado.

Apesar do acima descrito, alguns estudos parecem ser consensuais quanto à eficácia a curto prazo do extrato WS 5570, na dose de 900mg/dia, na depressão major ligeira a moderada (Diagnostic and Statistical Manual of Mental Disorders (DSM)-IV). ${ }^{4}$

Pese embora a evidência que favorece o potencial terapêutico do hipericão, será também de esperar a coexistência de um potencial para interações medicamentosas e reações adversas. ${ }^{14}$

Estas formulações de plantas medicinais podem ainda apresentar variabilidade qualitativa e quantitativa das substâncias ativas, ${ }^{14}$ aspeto que poderá ter relação com o risco de interações medicamentosas referido pela maioria dos estudos incluídos.

Por outro lado, alguns estudos descrevem a possibilidade de efeitos adversos associados ao uso do hipericão, ainda que a maioria não tenha procedido à des- crição dos mesmos, uma vez que não foram desenhados com esse objetivo. De notar que os efeitos adversos associados à sua utilização apenas foram mais prevalentes no grupo que utilizou hipericão, quando comparado com o grupo placebo, no estudo de Apaydin e colaboradores ${ }^{1} \mathrm{e}$ apenas nos potenciais efeitos neurológicos resultantes do uso desta substância.

Assim, após análise dos dados verificou-se a falta de evidência consistente acerca do perfil de segurança e ainda a ausência de autorização para o uso desta substância pelas entidades reguladoras. ${ }^{6}$ Surge então um problema que será tão mais preocupante quanto maior for o consumo destas substâncias de venda livre por parte dos indivíduos com maior risco de interações medicamentosas e/ou reações adversas (doentes crónicos, idosos ou polimedicados).

Relativamente às limitações do presente estudo, importa destacar a falta de consenso científico, algo que transparece pela disparidade entre metodologias adotadas, nomeadamente na ausência de conformidade quanto às doses, tipo de extratos de hipericão, escalas/métodos de avaliação de sintomatologia, classificação da PD (DSM-IV, DSM-5, International Classification of Diseases (ICD)-9 ou ICD-10) e pelos resultados contraditórios obtidos. Além disso, nem todos os estudos incluídos efetuaram avaliação a longo prazo do uso do hipericão, o que limita a sua recomendação nesse contexto.

Assim, torna-se necessária a realização de novos estudos, com maior uniformidade metodológica e que 
permitam a determinação da posologia adequada, bem como quais os doentes com PD que beneficiarão da integração do hipericão na sua terapêutica.

No futuro seria interessante proceder à comparação da eficácia do hipericão com a psicoterapia, bem como com outras medidas terapêuticas não farmacológicas convencionais no âmbito da PD major.

Concluindo, os dados disponíveis favorecem o uso de hipericão como uma opção terapêutica na PD major ligeira a moderada, ainda que constitua uma opção que carece de regulamentação pelas entidades competentes.

\section{REFERÊNCIAS BIBLIOGRÁFICAS}

1. Apaydin EA, Maher AR, Shanman R, Booth MS, Miles JN, Sorbero ME, et al. A systematic review of St. John's wort for major depressive disorder. Syst Rev. 2016;5(1):148.

2. Linde K, Kriston L, Rücker G, Jamil S, Schumann I, Meissner K, et al. Efficacy and acceptability of pharmacological treatments for depressive disorders in primary care: systematic review and network meta-analysis. Ann Fam Med. 2015;13(1):69-79.

3. Direção-Geral da Saúde. Portugal, Saúde mental em números - 2014. Lisboa: DGS; 2014.

4. Kasper S. Phytopharmaceutical treatment of anxiety, depression, and dementia in the elderly: evidence from randomized, controlled clinical trials. Wien Med Wochenschr. 2015;165(11-12):217-28.

5. Lantz MS, Buchalter E, Giambanco V. St. John's wort and antidepressant drug interactions in the elderly. J Geriatr Psychiatry Neurol. 1999;12(1):7-10.

6. Canedo F, Gamboa A. Hipericão: interacções medicamentosas. Bol Farmacovigilância. 2000;4(1):3-4.

7. Ernst E, Rand JI, Barnes J, Stevinson C. Adverse effects profile of the herbal antidepressant St. John's wort (Hypericum perforatum L.). Eur J Clin Pharmacol. 1998;54(8):589-94.

8. Observatório de Interações Planta-Medicamento. Erva de S. João /
Hipericão (Hypericum perforatum) [homepage]. Coimbra: Universidade de Coimbra; 2021. Available from: http://www.oipm.uc.pt/interacoes/index.php?target $=$ list\&search=plantas\&start_at $=20$

9. Ravindran AV, Balneaves LG, Faulkner G, Ortiz A, Mclntosh D, Morehouse RL, et al. Canadian Network for Mood and Anxiety Treatments (CANMAT) 2016 clinical guidelines for the management of adults with major depressive disorder: section 5. Complementary and alternative medicine treatments. Can J Psychiatry. 2016;61(9):576-87.

10. Scottish Intercollegiate Guidelines Network. Non-pharmaceutical management of depression in adults: a national clinical guideline [Internet]. Edinburgh: SIGN; 2010. Available from: https://www.ashlandmhrb.org/ upload/nonpharmaceutical_management_of_depression_in_adults__nhs_-_a_national_clinical_guideline.pdf

11. Sarris J. Herbal medicines in the treatment of psychiatric disorders: 10-year updated review. Phytother Res. 2018;32(7):1147-62.

12. Cipriani A, Barbui C, Butler R, Hatcher S, Geddes J. Depression in adults: drug and physical treatments. BMJ Clin Evid. 2011;2011:1003.

13. Eatemadnia A, Ansari S, Abedi P, Najar S. The effect of Hypericum perforatum on postmenopausal symptoms and depression: a randomized controlled trial. Complement Ther Med. 2019;45:109-13.

14. INFARMED. Plantas medicinais: interações medicamentosas. Bol Farmacovigilância. 2020;24(3):5-6.

\section{CONFLITO DE INTERESSES}

Os autores declaram não existir quaisquer conflitos de interesse.

\section{FINANCIAMENTO}

Os autores declaram não existir qualquer financiamento para a presente revisão.

\section{ENDEREÇO PARA CORRESPONDÊNCIA}

Tiago da Cunha Costa

E-mail: tiago_costa_25@hotmail.com

https://orcid.org/0000-0003-1110-1571

Recebido em 16-08-2020

Aceite para publicação em 17-07-2021 


\section{ABSTRACT}

\section{HYPERICUM IN DEPRESSIVE DISORDER: A CRITICAL REVIEW}

Objectives: Review the evidence regarding the effectiveness, tolerability, and safety of hypericum in the treatment of depressive disorder (DD).

Data sources: NICE, Canadian Medical Association Infobase, PubMed - Clinical Queries, The Cochrane Library, BMJ, Bandolier, and DARE - Centre for Reviews and Dissemination.

Methods: The research for scientific articles was performed on online Evidence-Based Medicine platforms, with the keywords 'Hypericum' and 'Depressive Disorder'. Articles were selected based on title, year of publication, and abstract.

Results: Two clinical guidelines (CG), three meta-analyses (MA), two systematic reviews (SR), and one original article (OA) were included in this review. One CG reported that hypericum can be used as first-line in mild to moderate major DD and as an adjuvant treatment of second-line in moderate to severe major DD. Another CG does not support the use. All of MA and SR conclude that hypericum is more effective than placebo in the treatment of DD, with two MA and two SR added that there is no increase of adverse effects. The two SR mentioned the risk of interactions with other drugs. The OA also affirms the effectiveness of hypericum in postmenopausal women.

Conclusion: There is evidence regarding the efficacy of hypericum in mild to moderate DD. The available data appears to be consistent with its good tolerability, but also in terms of the risk of interactions with other drugs. The limitations of the recommendation are essentially due to the mechanisms of action that are not fully understood.

Keywords: Hypericum; Depressive disorder. 